\title{
Understanding Academic Dishonesty: An Empirical Study in Two Undergraduate Business Colleges
}

\author{
Peggy Johnson \\ Lander University \\ Joseph Krupka \\ Florida State University \\ David Simmonds \\ Savannah State University
}

\begin{abstract}
The purpose of this study is to (i) identify the extent of student academic dishonesty in colleges of business at two AACSB accredited universities in the southeastern United States, (ii) empirically test a model of factors that influence student cheating, and (iii) explore recommendations for the faculty and administration of these institutions to reduce the incidence of academic dishonesty. A questionnaire was administered to business students in each college during spring semester 2019. Common factors that are explored in this study include individual behavior, situational vs planned behavior, environmental factors, and institutional components.
\end{abstract}

Keywords: Academic Dishonesty, Ethical Behavior, students

\section{INTRODUCTION}

Academic Dishonesty (AD) is any type of cheating that occurs in relation to a formal academic exercise. A large body of research dating back 70 years indicates that $\mathrm{AD}$ is pervasive in universities throughout the world. The prevalence of AD on college campuses in the United States has been reported that on average more than $70 \%$ of students have engaged in some form of AD (Whitley, 1998). An early study of cheating among business students by Bowers (1964), recorded higher levels of cheating among business students compared to students in other disciplines. McCabe and Trevino (1997) found similar differences in a study of 16 schools with science and engineering programs, that $84 \%$ of business students reporting one or more serious cheating incidents within the prior year compared to $72 \%$ of engineering students and $66 \%$ of all other participating students. Nearly all full-time faculty in one of the universities in this study reported numerous incidences of cheating each semester; this anecdotal evidence was the impetus for this study.

Prevalent factors that have been identified as having been empirically linked to AD include demographic characteristics, behavioral characteristics, situational factors and institutional factors. Demographic characteristics include factors such as age, gender, grade point average, and field of study. 
Behavioral characteristics include behaviors such as alcohol consumption, club membership, and participation in extracurricular activities. Situational factors include such things as peer behavior, class size, course delivery methods, instructor surveillance and institutional factors such as honor codes. One of the most important determinants of student cheating is the belief or perception that everyone else is already cheating, "An institution's ability to develop a shared understanding and acceptance of its academic integrity policies has a significant and substantive impact on student perception of their peer's behavior, the most powerful influence on self-reported cheating." (McCabe and Trevino, 1993)

Reviews of factors related to cheating have been studied and identified by (Crown and Spiller, 1998); (McCabe, Trevino, and Butterfield, 2001); and (Simha and Cullen, 2012). The large multi-institutional studies of the attitudes of students concerning cheating have recognized that the factors mentioned here have been established as playing a critical role regarding the propensity of students to cheat. However, the newest situational factor, the dissemination of information technology has not been studied systematically to the degree previous learning environments have. It is widely accepted that information technology has lowered barriers to cheating according to Etter, Cramer, and Finn (2006). Previous studies posit that cheating occurs when opportunities are enhanced (Michaels \& Miethe, 1989; Perry, Kane, Bernesser, \& Spicker, 1990). Additionally, anecdotal evidence indicates that information technology allows students to participate in $\mathrm{AD}$ to a greater degree than has been previously recognized. This study investigates both inclass and on-line cheating among participants.

Previous research (Jackson, Levine, Furham, \& Burr, 2002; Whitley, 1998) indicates that students who are more likely to engage in $\mathrm{AD}$ in a university setting are male, young (Klein, Levenburg, McKendall, \& Mothersel, 2007) and members of fraternities or sororities ( Baird, 1980; Burrus, McGoldrick, \& Schuhmann, 2007). They also have a tendency to consume alcohol, are more likely to cheat if they believe their peers cheat, and have low grade point averages (Wray, Jones, Schuhmann, and Burrus, 2016).

A considerable amount of the data collected in prior research has been self-reported by students, this data is easy to obtain but may result in underestimation of the actual cheating due to the lack of incentive for honesty and the fear of reprisal (Wray, Jones, Schuhmann, and Burrus, 2016). After the data is collected, the usual methods of analysis such as statistical tests for differences in cheating between groups (i.e. Accounting majors vs Marketing majors) along with tests for differences in characteristics of students (i.e. age, gender, grade-point average) between students who cheat and those who do not will be employed. Examples of these statistical tests include t-tests for differences in means, chi-square tests for differences in proportions or analysis of (co) variance, additionally correlation and regression analysis will be employed to assess factors associated with cheating.

The Theory of Planned Behavior (TPB) started as the Theory of Reasoned Action in 1980 to predict an individual's intention to engage in a behavior at a specific time and place. The theory was intended to explain all behaviors over which people have the ability to exert self-control. The key component to this model is behavioral intent; behavioral intentions are influenced by the attitude about the likelihood that the behavior will have the expected outcome and the subjective evaluation of the risks and benefits of that outcome. Behavioral intention refers to the motivational factors that influence a given behavior where the stronger the intention to perform the behavior, the more likely the behavior will be performed.

The TPB has been used successfully to predict and explain a wide range of health behaviors and intentions including smoking, drinking, health services utilization, breastfeeding, and substance use, among others. The TPB states that behavioral achievement depends on both motivation (intention) and ability (behavioral control). It distinguishes between three types of beliefs - behavioral, normative, and control. The TPB is comprised of six constructs that collectively represent a person's actual control over the behavior.

The combined total surveys completed by the students from both universities was 571 . There were 351 completed surveys from one university and 221 from the other. We conducted analysis of variance and regression analysis using the dependent variables of intention to cheat this term and will cheat given the opportunity. The independent variables include: I have the skills to cheat, cheating is not morally 
wrong, I cheated during the previous term, my support source is scholarships, and love ones expect cheating.

\section{DEMOGRAPHIC FACTORS}

The demographic factors that stood out from our survey are shown below.

\section{FIGURE 1 \\ SCHOLARSHIP SUPPORT}

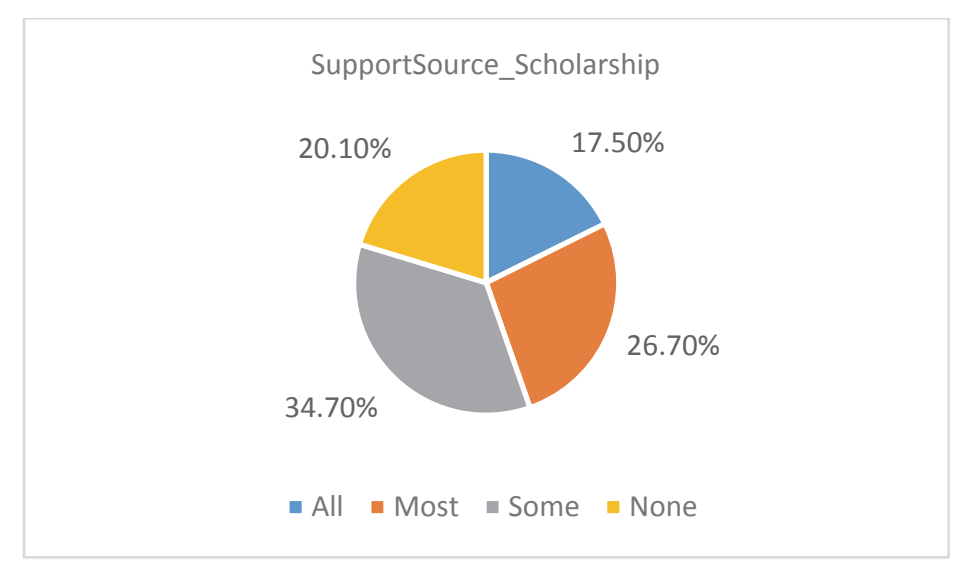

$44.2 \%$ of the sample were receiving either full or mostly scholarships to fund their financial support. Meaning scholarships have an important impact on the funding for our sample.

FIGURE 2

PARENTS/FAMILY SUPPORT

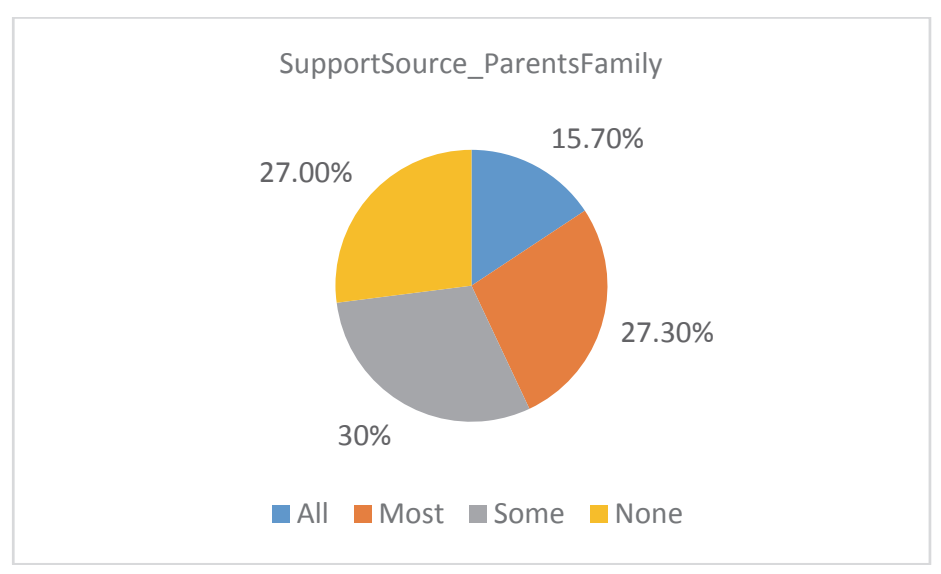

$43 \%$ of the responses showed parental support either in full or mostly. Only $27 \%$ did not receive any parental financial support. 
FIGURE 3

\section{SUPPORT MYSELF}

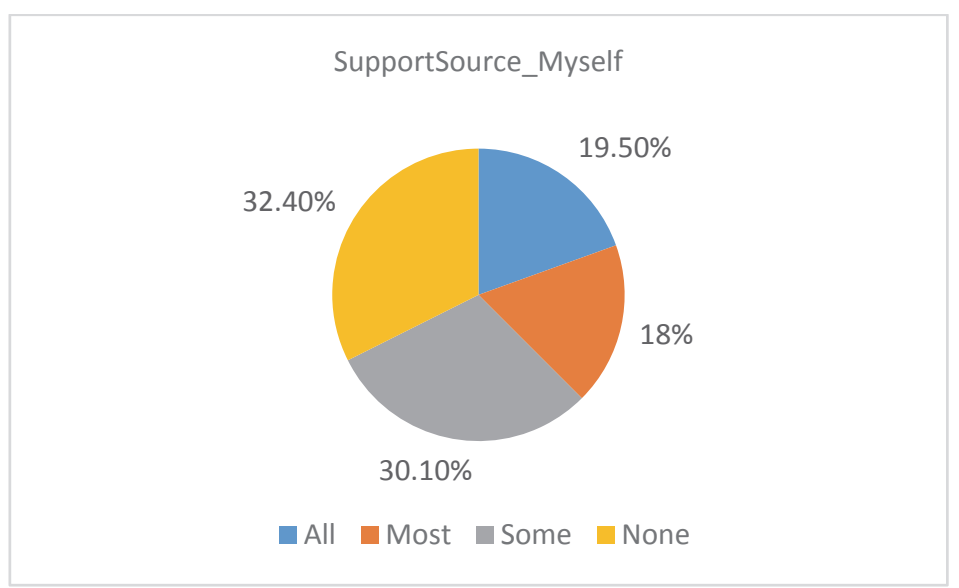

$67.6 \%$ of the students contributed at least some to their own financial support. $32.4 \%$ responded that they did not contribute at all to their financial support receiving all their support from others such as parental or scholarship support.

FIGURE 4 SUPPORT BY OTHERS

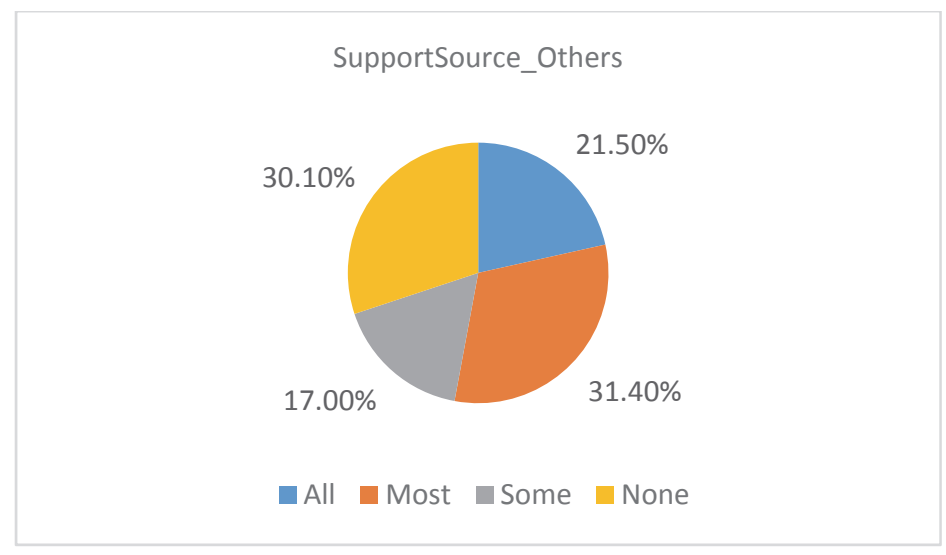

$69.9 \%$ of the students received some financial support from others. 
FIGURE 5

YEAR IN SCHOOL

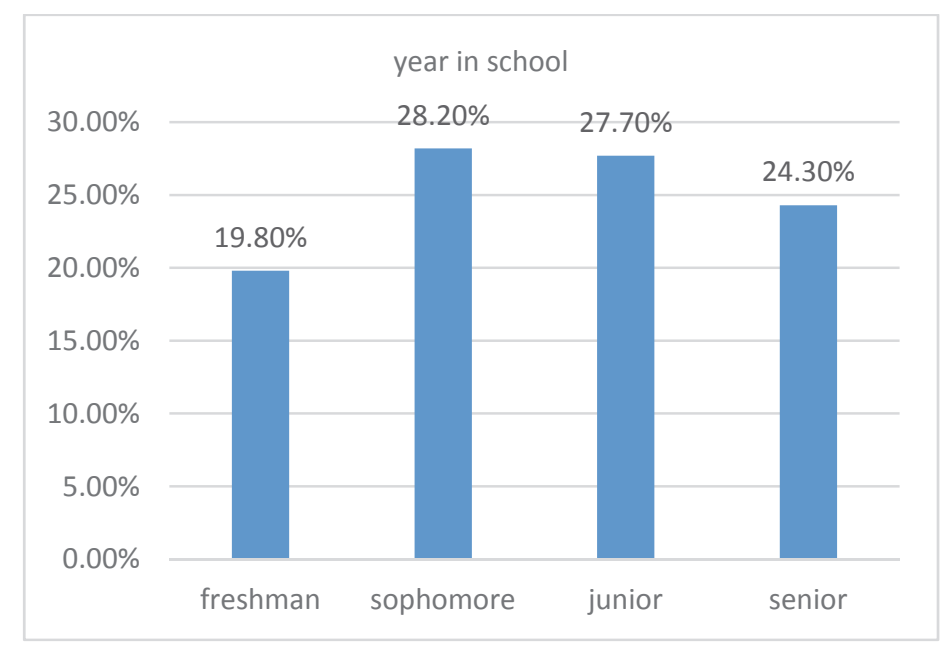

Our sample was immensely blended with students from each year in school. This helped gather substantial data from students in all four years in school.

FIGURE 6

GENDER

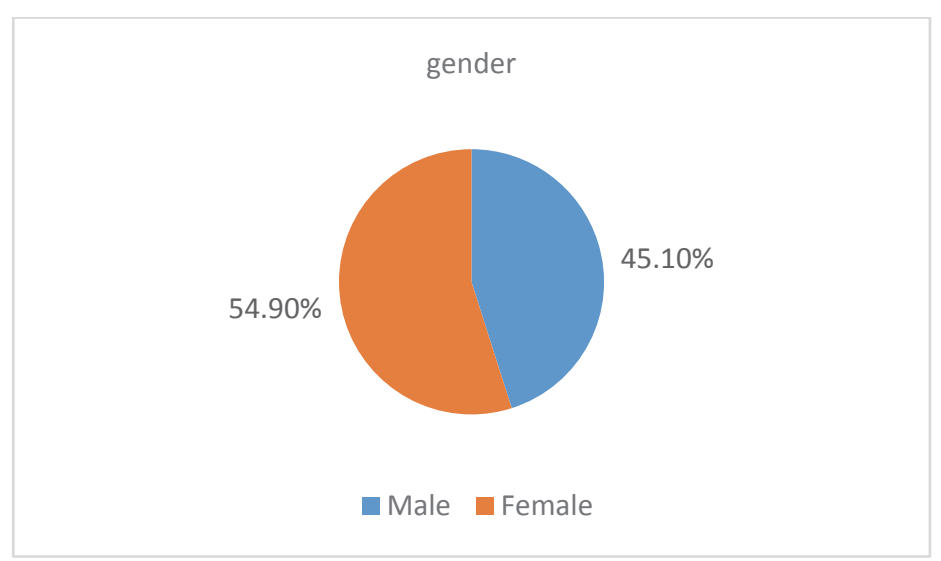

The mix between male and female students were $55.9 \%$ female and $45.1 \%$ male. Again, this demographic contributed a solid gender mix in our sample. 


\section{FIGURE7}

MAJOR

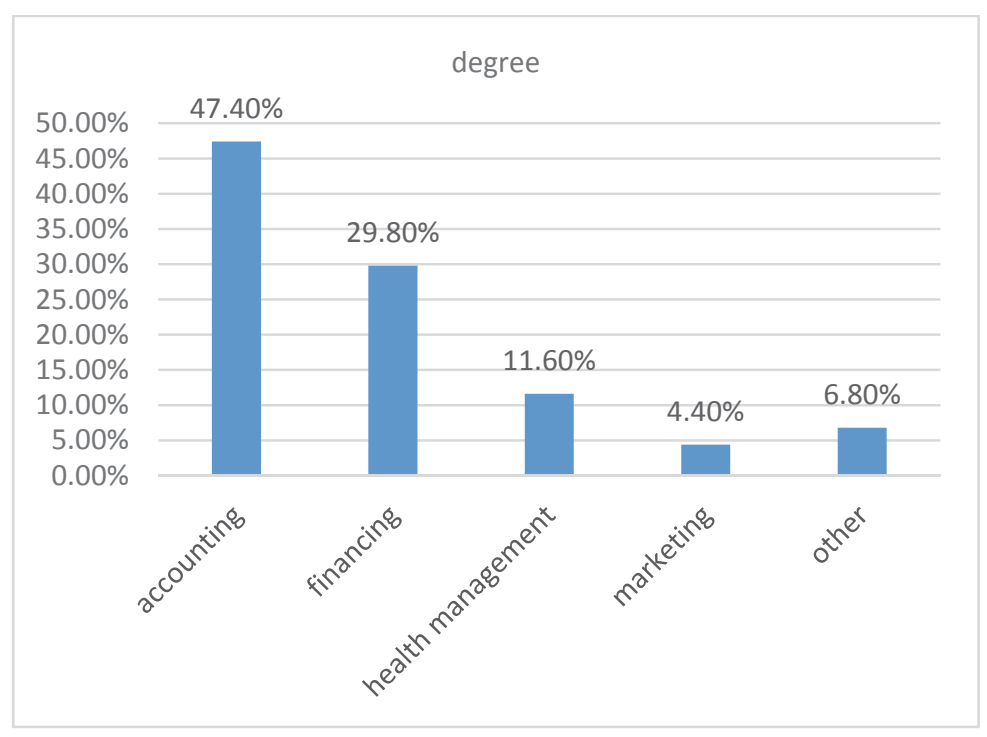

$47.4 \%$, almost half of our sample were accounting majors. The other noteworthy major from our sample was finance which was $29.8 \%$ of the sample.

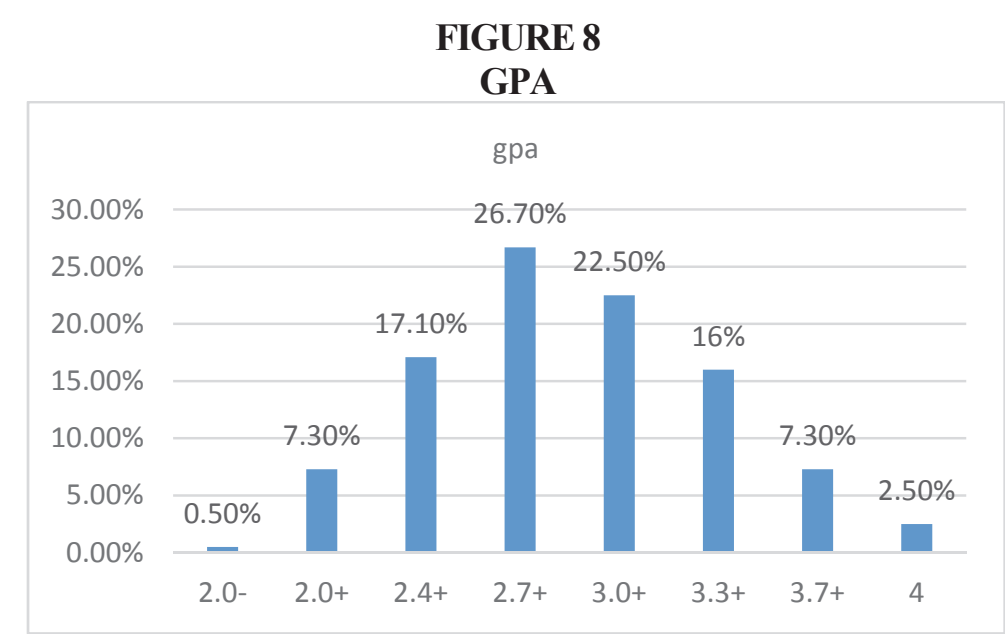

Our sample showed a true bell curve of GPA's. We gathered data from all sides of the GPA spectrum.

\section{HYPOTHESES}

H1a: I_Have_SkillsTo_Cheat will positively affect WillCheat_GivenOpportunity VS

H1b: I_Have_SkillsTo_Cheat will positively affect IntendTo_Cheat_ThisTerm

H2a: Cheating_NotMorallyWrong will positively affect WillCheat_GivenOpportunity

H2b: Cheating_NotMorallyWrong will positively affect Intend To CheatThisTerm

H3a: LovedOnes_Expect_Cheating will positively affect WillCheat_GivenOpportunity

H3b: LovedOnes_Expect_Cheating will positively affect IntendTo_Cheat_ThisTerm 
H4a: SupportSource_Myself will positively affect WillCheat_GivenOpportunity

H4b: SupportSource_Myself will positively affect IntendTo_Cheat_ThisTerm

H5a: SupportSource_Scholarship will positively affect WillCheat_GivenOpportunity

H5b: SupportSource_Scholarship will positively affect IntendTo_Cheat_ThisTerm

H6a: PreviousTerm_FrequencyCheating_HW will positively affect WillCheat_GivenOpportunity

H6b: PreviousTerm_FrequencyCheating_HW will positively affect IntendTo_Cheat_ThisTerm

\section{METHODOLOGY}

\section{Model 1- WillCheat_GivenOpportunity VS I_Have_SkillsTo_Cheat}

The actual behavior control of an individual, according to the theory of planned behavior, depends on the extent to which a person has the skills, resources, and other prerequisites needed to perform a behavior. Skill sets are both an asset and an investment. Skills to cheat are like any other skill, the perception of having them makes it more likely an individual will use them according to past studies (Atmeh \& Khadash, 2008). So, whether their skills to cheat are real or perceived, the more skills they have, the more likely they are to cheat. Again, we see no difference between intentional and opportunistic cheating. Skills to cheat drives them both.

\section{MODEL-1a WILLCHEAT_GIVENOPPORTUNITY VS I_HAVE_SKILLSTO_CHEAT}

\begin{tabular}{|c|c|c|c|c|}
\hline Independent Variables & Beta & $\mathbf{t}$ & p-value & \\
\hline Year2Transfer & -0.0038 & -0.015 & 0.9877 & \\
\hline Year4Transfer & 0.3649 & 1.08 & 0.2811 & \\
\hline International & 0.5333 & 1.817 & 0.0702 & . \\
\hline Gender & 0.2765 & 2.041 & 0.0421 & $*$ \\
\hline I_Have_SkillsTo_Cheat & 0.4022 & 7.361 & $1.4 \mathrm{E}-12$ & $* * *$ \\
\hline f-statistic $=16.35$ & & & & \\
\hline $\mathbf{R}^{2}=0.1961$ & & & & \\
\hline $\operatorname{adj}-R^{2}=0.1841$ & & & & \\
\hline $\mathbf{p}=1.945 \mathrm{e}-14$ & & & & \\
\hline$* \boldsymbol{p}<0.05, * * \boldsymbol{p}<0.01, * * * \boldsymbol{p}<0.001$ & & & & \\
\hline
\end{tabular}

Hypothesis-1a suggested that higher levels of I_Have_SkillsTo_Cheat would result in increased WillCheat_GivenOpportunity. Overall, model-N was significant $(\mathbf{f}=16.35, \mathbf{p}=1.945 \mathrm{e}-14$. $)$

And Hypothesis-1a was supported since I_Have_SkillsTo_Cheat was significant in the regression against WillCheat_GivenOpportunity $(\boldsymbol{\beta}=0.4022, \mathbf{t}=7.361, \mathbf{p}=1.425 \mathrm{e}-12)$. 
MODEL-1b

INTENDTO_CHEAT_THISTERM VS I_HAVE_SKILLSTO_CHEAT

\begin{tabular}{|c|c|c|c|c|}
\hline Independent Variables & Beta & $\mathbf{t}$ & p-value & \\
\hline Year2Transfer & 0.2824 & 1.634 & 0.103 & \\
\hline Year4Transfer & -0.1790 & -0.745 & 0.457 & \\
\hline international & 0.1645 & 0.788 & 0.431 & \\
\hline Gender & 0.4091 & 4.249 & $2.8 \mathrm{E}-05$ & $* * *$ \\
\hline I_Have_SkillsTo_Cheat & 0.4271 & 11.019 & $<2 \mathrm{E}-16$ & $* * *$ \\
\hline \multicolumn{5}{|l|}{ f-statistic $=32.65$} \\
\hline \multicolumn{5}{|l|}{$\mathbf{R}^{2}=0.327$} \\
\hline \multicolumn{5}{|l|}{$\operatorname{adj}-\mathbf{R}^{2}=0.317$} \\
\hline \multicolumn{5}{|l|}{$\mathbf{p}=4.052 \mathrm{e}-27$} \\
\hline$* \boldsymbol{p}<0.05, * * \boldsymbol{p}<0.01, * * * \boldsymbol{p}<0.001$ & & & & \\
\hline
\end{tabular}

Hypothesis-1b: suggested that higher levels of I_Have_SkillsTo_Cheat would result in increased IntendTo_Cheat_ThisTerm. Overall, model-Nb was significant $(\mathbf{f}=32 . \overline{6} 5, \mathbf{p}=4.052 \mathrm{e}-27)$.

And Hypothesis-1b was supported since I_Have_SkillsTo_Cheat was significant in the regression against IntendTo_Cheat_ThisTerm $(\boldsymbol{\beta}=0.4271, \mathbf{t}=11.02, \overline{\mathbf{p}}=2.608 \mathrm{e}-24)$.

\section{Model 2-WillCheat_GivenOpportunity VS Cheating_NotMorallyWrong}

Moral obligation as a predictor of academic dishonesty has been demonstrated in several studies (Harding et al., 2007; Whitley, 1998). Beck and Ajzen (1991) showed that moral that moral obligation was a precursor of intent to cheat and also a significant predictor of actual cheating behavior. Since our actions are a combination of motivation (being a better grade with less effort here), and inhibition (being the possibly of being caught as well as guilt over the immorality), if one of the inhibitions are removed, then the student may be more likely to carry out acts of cheating. Guilt about doing immoral acts is certainly one of the checks and balances which prevent immoral behavior. Removing the moral factor makes it all about the temptation (which the large majority of people will feel) against only the prospect of getting caught. Again both sides of the hypothesis were supported showing that immorality will allow people to cheat given the opportunity, and also cause them to plan to cheat. 


\section{MODEL-2a}

WILLCHEAT_GIVENOPPORTUNITY VS CHEATING_NOTMORALLYWRONG

\begin{tabular}{|c|c|c|c|c|}
\hline Independent Variables & Beta & $\mathbf{t}$ & p-value & \\
\hline Year2Transfer & -0.0108 & -0.044 & 0.9651 & \\
\hline Year4Transfer & 0.5377 & 1.588 & 0.1132 & \\
\hline International & 0.6874 & 2.087 & 0.0376 & $*$ \\
\hline Gender & 0.2435 & 1.769 & 0.0777 & . \\
\hline Cheating_NotMorallyWrong & 0.4025 & 7.382 & $1.3 \mathrm{E}-12$ & $* * *$ \\
\hline f-statistic $=15.67$ & & & & \\
\hline $\mathbf{R}^{2}=0.1923$ & & & & \\
\hline adj- $R^{2}=0.1801$ & & & & \\
\hline $\mathbf{p}=7.714 \mathrm{e}-14$ & & & & \\
\hline${ }^{*} \boldsymbol{p}<0.05, * * \boldsymbol{p}<0.01, * * * \boldsymbol{p}<0.001$ & & & & \\
\hline
\end{tabular}

Hypothesis-2a suggested that higher levels of Cheating_NotMorallyWrong would result in increased WillCheat_GivenOpportunity. Overall, model-N was significant $(\mathbf{f}=15.67, \mathbf{p}=7.714 \mathrm{e}-14)$.

And Hypothesis-2a was supported since Cheating_NotMorallyWrong was significant in the regression against WillCheat_GivenOpportunity $(\boldsymbol{\beta}=0.4025, \mathbf{t}=7.382, \mathbf{p}=1.284 \mathrm{e}-12$.

\section{MODEL-2b \\ INTENDTO_CHEAT_THISTERM VS CHEATING_NOTMORALLYWRONG}

\begin{tabular}{|c|c|c|c|c|}
\hline Independent Variables & Beta & $\mathbf{t}$ & p-value & \\
\hline Year2Transfer & 0.2785 & 1.443 & 0.15003 & \\
\hline Year4Transfer & 0.1482 & 0.558 & 0.57723 & \\
\hline International & 0.4391 & 1.699 & 0.09023 & . \\
\hline Gender & 0.4207 & 3.904 & 0.00012 & $* * *$ \\
\hline Cheating_NotMorallyWrong & 0.2686 & 6.332 & $7.9 \mathrm{E}-10$ & $* * *$ \\
\hline \multicolumn{5}{|l|}{ f-statistic $=14.52$} \\
\hline \multicolumn{5}{|l|}{$\mathbf{R}^{2}=0.1803$} \\
\hline \multicolumn{5}{|l|}{ adj-R $\mathbf{R}^{2}=0.1679$} \\
\hline \multicolumn{5}{|l|}{$\mathbf{p}=7.275 \mathrm{e}-13$} \\
\hline${ }^{*} \boldsymbol{p}<0.05, * * \boldsymbol{p}<0.01, * * * \boldsymbol{p}<0.001$ & & & & \\
\hline
\end{tabular}

Hypothesis-2b suggested that higher levels of Cheating_NotMorallyWrong would result in increased IntendTo_Cheat_ThisTerm. Overall, model-N was significant $(\mathbf{f}=14.52, \mathbf{p}=7.275 \mathrm{e}-13)$.

And Hypothesis-2b was supported since Cheating_NotMorallyWrong was significant in the regression against IntendTo_Cheat_ThisTerm $(\boldsymbol{\beta}=0.2686, \mathbf{t}=6.332, \mathbf{p}=7.89 \mathrm{e}-10)$. 


\section{Model 3- WillCheat_GivenOpportunity VS LovedOnes_Expect_Cheating}

Subjective norms have been found to be a key factor in the model for determining behavioral intentions (Alleyne \& Phillips). They found that the subjective norms had an impact on the student's attitudes toward academic dishonesty. In measuring subjective norms with respect to academic dishonesty was measured by using the following questions, 1) People whose opinions I value (e.g. my family, friends, colleagues, teachers, etc.) expect me to cheat on a test or exam. 2) Most people who are important to me (e.g. my family, friends, colleagues, teachers, etc.) will look down on me if I cheat on an exam or assignment. Prior studies also found support that attitudes of students and the influence of significant others and peers would endorse the practice of academic dishonesty (Chapman et al, 2004).

\section{MODEL-3a WILLCHEAT_GIVENOPPORTUNITY VS LOVEDONES_EXPECT_CHEATING}

\begin{tabular}{|c|c|c|c|c|}
\hline Independent Variables & Beta & $\mathbf{t}$ & p-value & \\
\hline Year2Transfer & -0.0807 & -0.337 & 0.7362 & \\
\hline Year4Transfer & 0.5421 & 1.651 & 0.0996 & \\
\hline international & 0.8714 & 3.1 & 0.0021 & $* *$ \\
\hline Gender & 0.1021 & 0.749 & 0.4541 & \\
\hline LovedOnes_Expect_Cheating & 0.5049 & 8.135 & $8.1 \mathrm{E}-15$ & $* * *$ \\
\hline f-statistic $=18.92$ & & & & \\
\hline $\mathbf{R}^{2}=0.2202$ & & & & \\
\hline adj- $R^{2}=0.2085$ & & & & \\
\hline $\mathbf{p}=1.424 \mathrm{e}-16$ & & & & \\
\hline$* \boldsymbol{p}<0.05, * * \boldsymbol{p}<0.01, * * * \boldsymbol{p}<0.001$ & & & & \\
\hline
\end{tabular}

Hypothesis-3a suggested that higher levels of LovedOnes_Expect_Cheating would result in increased WillCheat_GivenOpportunity. Overall, model-N was significant $(\mathbf{f}=18.92, \mathbf{p}=1.424 \mathrm{e}-16)$.

And Hypothesis-3a was supported since LovedOnes_Expect_Cheating was significant in the regression against WillCheat_GivenOpportunity $(\boldsymbol{\beta}=0.5049, \mathbf{t}=8 . \overline{1} 35, \mathbf{p}=\overline{8} .125 \mathrm{e}-15)$. 
MODEL-3b

INTENDTO_CHEAT_THISTERM VS LOVEDONES_EXPECT_CHEATING

\begin{tabular}{|c|c|c|c|c|}
\hline Independent Variables & Beta & $\mathbf{t}$ & p-value & \\
\hline Year2Transfer & 0.2069 & 1.188 & 0.23556 & \\
\hline Year4Transfer & 0.0012 & 0.005 & 0.99587 & \\
\hline international & 0.5490 & 2.687 & 0.00756 & $* *$ \\
\hline Gender & 0.2476 & 2.516 & 0.01235 & $*$ \\
\hline LovedOnes_Expect_Cheating & 0.4915 & 11.024 & $<2 \mathrm{E}-16$ & $* * *$ \\
\hline \multicolumn{5}{|l|}{ f-statistic $=32.57$} \\
\hline \multicolumn{5}{|l|}{$\mathbf{R}^{2}=0.3252$} \\
\hline \multicolumn{5}{|l|}{ adj- $\mathbf{R}^{2}=0.3152$} \\
\hline \multicolumn{5}{|l|}{$\mathbf{p}=4.294 \mathrm{e}-27$} \\
\hline$* \boldsymbol{p}<0.05, * * \boldsymbol{p}<0.01, * * * \boldsymbol{p}<0.001$ & & & & \\
\hline
\end{tabular}

Hypothesis-3b suggested that higher levels of LovedOnes_Expect_Cheating would result in increased IntendTo_Cheat_ThisTerm. Overall, model-Nb was significant $(\mathbf{f}=32.57, \mathbf{p}=4.294 \mathrm{e}-27)$.

And Hypothesis-3b was supported since LovedOnes_Expect_Cheating was significant in the regression against IntendTo_Cheat_ThisTerm $(\boldsymbol{\beta}=0.4915, \mathbf{t}=11.0 \overline{2}, \mathbf{p}=2.396 \mathrm{e}-24)$.

\section{Model 4- WillCheat_GivenOpportunity VS SupportSource_Myself}

Many students, both graduate and undergraduate work while attending school today, which according to prior research increases the pressures on them to perform well. (Macabe, et al, 2006). Finally, many business students have less time to study and complete assignments, and graduate students may be under pressure to keep their grades up in order to continue getting financial support from their employers.

\section{MODEL-4a \\ WILLCHEAT_GIVENOPPORTUNITY VS SUPPORTSOURCE_MYSELF}

\begin{tabular}{|c|c|c|c|c|}
\hline Independent Variables & Beta & $\mathbf{t}$ & p-value & \\
\hline Year2Transfer & -0.1143 & -0.376 & 0.70715 & \\
\hline Year4Transfer & 1.1100 & 3.035 & 0.00263 & $* *$ \\
\hline international & 0.9661 & 2.689 & 0.00759 & $* *$ \\
\hline Gender & 0.1254 & 0.787 & 0.43166 & \\
\hline SupportSource_Myself & 0.0682 & 1.052 & 0.29391 & \\
\hline \multicolumn{5}{|l|}{ f-statistic $=4.046$} \\
\hline \multicolumn{5}{|l|}{$\mathbf{R}^{2}=0.06585$} \\
\hline \multicolumn{5}{|l|}{ adj- $\mathbf{R}^{2}=0.04957$} \\
\hline $\mathbf{p}=0.001453$ & & & & \\
\hline
\end{tabular}


Hypothesis-4a suggested that higher levels of SupportSource_Myself would result in increased WillCheat_GivenOpportunity. Overall, model-N was significant $(\mathbf{f}=4.046, \mathbf{p}=0.001453)$.

But Hypothesis-4a was not supported since SupportSource_Myself was not significant in the regression against WillCheat_GivenOpportunity $(\boldsymbol{\beta}=0.06822, \mathbf{t}=1.052, \mathbf{p}=0.2939)$.

\section{MODEL-4b
INTENDTO_CHEAT_THISTERM VS SUPPORTSOURCE_MYSELF}

\begin{tabular}{|c|c|c|c|c|}
\hline Independent Variables & Beta & $\mathbf{t}$ & p-value & \\
\hline Year2Transfer & 0.3963 & 1.753 & 0.08073 & . \\
\hline Year4Transfer & 0.4069 & 1.548 & 0.12274 & \\
\hline international & 0.8318 & 3.113 & 0.00204 & $* *$ \\
\hline Gender & 0.2564 & 2.168 & 0.03097 & $*$ \\
\hline SupportSource_Myself & 0.1854 & 3.839 & 0.00015 & $* * *$ \\
\hline \multicolumn{5}{|l|}{ f-statistic $=6.708$} \\
\hline \multicolumn{5}{|l|}{$\mathbf{R}^{2}=0.1043$} \\
\hline \multicolumn{5}{|l|}{ adj- $R^{2}=0.08876$} \\
\hline \multicolumn{5}{|l|}{$\mathbf{p}=6.248 \mathrm{e}-06$} \\
\hline${ }^{*} \boldsymbol{p}<0.05, * * p<0.01, * * * p<0.001$ & & & & \\
\hline
\end{tabular}

Hypothesis-4b suggested that higher levels of SupportSource_Myself would result in increased IntendTo_Cheat_ThisTerm. Overall, model-N was significant $(\mathbf{f}=6.708, \mathbf{p}=6.248 \mathrm{e}-06)$.

And Hypothesis-4b was supported since SupportSource_Myself was significant in the regression against IntendTo_Cheat_ThisTerm $(\boldsymbol{\beta}=0.1853, \mathbf{t}=3.839, \mathbf{p}=0.000152)$.

Model 5 - SupportSource_Scholarship will positively affect WillCheat_GivenOpportunity

MODEL-5a

WILLCHEAT_GIVENOPPORTUNITY VS SUPPORTSOURCE_SCHOLARSHIP

\begin{tabular}{|c|c|c|c|c|}
\hline Independent Variables & Beta & $\mathbf{t}$ & p-value & \\
\hline Year2Transfer & -0.1804 & -0.642 & 0.52154 & \\
\hline Year4Transfer & 0.8359 & 2.375 & 0.01815 & $*$ \\
\hline international & 1.1059 & 3.549 & 0.00045 & $* * *$ \\
\hline Gender & 0.2386 & 1.573 & 0.11684 & \\
\hline SupportSource_Scholarship & 0.1185 & -1.642 & 0.1016 & \\
\hline \multicolumn{5}{|l|}{ f-statistic $=4.546$} \\
\hline \multicolumn{5}{|l|}{$\mathbf{R}^{2}=0.06956$} \\
\hline \multicolumn{5}{|l|}{ adj- $R^{2}=0.05426$} \\
\hline \multicolumn{5}{|l|}{$\mathbf{p}=0.0005176$} \\
\hline$* \boldsymbol{p}<0.05, * * \boldsymbol{p}<0.01, * * * \boldsymbol{p}<0.001$ & & & & \\
\hline
\end{tabular}


Hypothesis-5a suggested that higher levels of SupportSource_Scholarship would result in increased WillCheat_GivenOpportunity. Overall, model-N was significant $(\mathbf{f}=4.546, \mathbf{p}=0.0005176)$.

But Hypothesis-5a was not supported since SupportSource_Scholarship was not significant in the regression against WillCheat_GivenOpportunity $(\boldsymbol{\beta}=0.1185, \mathbf{t}=-1.642, \mathbf{p}=0.1016)$.

\section{INTENDTO_CHEAT_THISTERM VS SUPPORTSOURCE_SCHOLARSHIP}

\begin{tabular}{|c|c|c|c|c|}
\hline Independent Variables & Beta & $\mathbf{t}$ & p-value & \\
\hline Year2Transfer & 0.1446 & 0.713 & 0.47665 & \\
\hline Year4Transfer & 0.3228 & 1.27 & 0.20497 & \\
\hline international & 0.7378 & 3.279 & 0.00116 & $* *$ \\
\hline Gender & 0.3406 & 3.113 & 0.00203 & $* *$ \\
\hline SupportSource_Scholarship & 0.2130 & -4.107 & $5.2 \mathrm{E}-05$ & $* * *$ \\
\hline \multicolumn{5}{|l|}{ f-statistic $=7.697$} \\
\hline \multicolumn{5}{|l|}{$\mathbf{R}^{\mathbf{2}}=0.112$} \\
\hline \multicolumn{5}{|l|}{ adj-R $\mathbf{R}^{2}=0.09749$} \\
\hline \multicolumn{5}{|l|}{$\mathbf{p}=7.828 \mathrm{e}-07$} \\
\hline${ }^{*} \boldsymbol{p}<0.05, * * \boldsymbol{p}<0.01, * * * \boldsymbol{p}<0.001$ & & & & \\
\hline
\end{tabular}

Hypothesis-5b: suggested that higher levels of SupportSource_Scholarship would result in increased IntendTo_Cheat_ThisTerm. Overall, model-N was significant $(\mathbf{f}=\overline{7} .697, \mathbf{p}=7.828 \mathrm{e}-07)$.

And Hypothesis-5b: was supported since SupportSource_Scholarship was significant in the regression against IntendTo_Cheat_ThisTerm $(\boldsymbol{\beta}=0.213, \mathbf{t}=-4.107, \mathbf{p}=5.146 \mathrm{e}-05)$. Again we see a difference between these two sides of this hypothesis since scholarship-support drives intention to cheat but not opportunistic cheating. Again, suggesting that with a scholarship at stake, the student is more likely to cheat and will not leave it to chance, since there is a lot riding on it. The consistency of support for the intention side versus the opportunistic side means students who support themselves or are supported through scholarships put their game face on when it comes to cheating and will not be easily detected since they come prepared. 
MODEL-6a

WILLCHEAT_GIVENOPPORTUNITY VS PREVIOUSTERM_FREQUENCYCHEATING_HW

\begin{tabular}{|c|c|c|c|c|}
\hline Independent Variables & Beta & $\mathbf{t}$ & p-value & \\
\hline Year2Transfer & 0.1348 & 0.528 & 0.59814 & \\
\hline Year4Transfer & 0.9380 & 2.716 & 0.00694 & $* *$ \\
\hline international & 1.0424 & 3.513 & 0.00051 & $* * *$ \\
\hline Gender & 0.3472 & 2.458 & 0.01449 & $*$ \\
\hline PreviousTerm_FrequencyCheating_HW & 0.4056 & 4.71 & $3.6 \mathrm{E}-06$ & $* * *$ \\
\hline \multicolumn{5}{|l|}{ f-statistic $=9.496$} \\
\hline \multicolumn{5}{|l|}{$\mathbf{R}^{2}=0.1241$} \\
\hline \multicolumn{5}{|l|}{ adj- $\mathbf{R}^{2}=0.1111$} \\
\hline \multicolumn{5}{|l|}{$\mathbf{p}=1.749 \mathrm{e}-08$} \\
\hline$* p<0.05, * * p<0.01, * * * p<0.001$ & & & & \\
\hline
\end{tabular}

Hypothesis-6a suggested that higher levels of PreviousTerm_FrequencyCheating_HW would result in increased WillCheat_GivenOpportunity. Overall, model-N was significant $(\mathbf{f}=9.496, \mathbf{p}=1.749 \mathrm{e}-08)$.

And Hypothesis-6a was supported since PreviousTerm_FrequencyCheating_HW was significant in the regression against WillCheat_GivenOpportunity $(\boldsymbol{\beta}=0.40 \overline{5} 6, \mathbf{t}=4.71, \mathbf{p}=3.628 \mathrm{e}-06)$.

\section{MODEL-6b \\ INTENDTO_CHEAT_THISTERM VS PREVIOUSTERM_FREQUENCYCHEATING_HW}

\begin{tabular}{|c|c|c|c|c|}
\hline Independent Variables & Beta & $\mathbf{t}$ & p-value & \\
\hline Year2Transfer & 0.3582 & 1.784 & 0.07536 & \\
\hline Year4Transfer & 0.2823 & 1.075 & 0.28324 & \\
\hline international & 0.7409 & 3.176 & 0.00163 & $* *$ \\
\hline Gender & 0.4727 & 4.278 & $2.5 \mathrm{E}-05$ & $* * *$ \\
\hline PreviousTerm_FrequencyCheating_HW & 0.2270 & 3.403 & 0.00075 & $* * *$ \\
\hline \multicolumn{5}{|l|}{ f-statistic $=8.607$} \\
\hline \multicolumn{5}{|l|}{$\mathbf{R}^{\mathbf{2}}=0.1129$} \\
\hline \multicolumn{5}{|l|}{ adj- $R^{2}=0.09982$} \\
\hline \multicolumn{5}{|l|}{$\mathbf{p}=1.087 \mathrm{e}-07$} \\
\hline${ }^{*} \boldsymbol{p}<0.05, * * p<0.01, * * * p<0.001$ & & & & \\
\hline
\end{tabular}

Hypothesis-6b suggested that higher levels of PreviousTerm_FrequencyCheating_HW would result in increased IntendTo_Cheat_ThisTerm. Overall, model-N was significant $(\mathbf{f}=8.607, \mathbf{p}=1.087 \mathrm{e}-07)$.

And Hypothesis-6b was supported since PreviousTerm_FrequencyCheating_HW was significant in the regression against IntendTo_Cheat_ThisTerm $(\boldsymbol{\beta}=0.227, \mathbf{t}=3.403, \mathbf{p}=0.00075)$. 
The fact that both intentions to cheat and cheating given opportunity is not surprising, since more time is spent on homework. So, if students cheat on homework, in which they have more time, can get guidance and have all the resources (literally in the world) at their disposal, then it is a part of their work ethic. And certainly, must be considered a part of their considered behavior, happening at a higher, planned behavior level than happens when they party their life away and suddenly find themselves with too little time to study to get a good grade for an exam. And so, it is to be expected that if they cheat on homework, they will certainly intend to cheat. And naturally, they will also cheat given the opportunity.

\section{ANALYSIS}

Hypotheses were tested using Ordinary Least Squares (OLS) regression in R. All models included Year-2-Transfers, Year-4-Transfers and International-student as control variables.

\section{Dependent Variables}

Dependent Variables for all models were IntendTo_Cheat_ThisTerm \& WillCheat_GivenOpportunity

\section{Independent Variables}

Dependent Variables were: I_Have_SkillsTo_Cheat, Cheating_NotMorallyWrong, LovedOnes_Expect_Cheating, SupportSource_Myself, SupportSource_Scholarship,

\section{Data Collection}

Data was collected by Survey data which had 40 questions including 15 Demographic Questions. The questionnaire was distributed at both universities during the Spring semester of 2019. Several Faculty members were enlisted to collect the survey data during their classes at the two universities. There was a total of 571 surveys completed, 350 completed surveys collected from one university, while 221 results were collected from the other.

\section{DISCUSSION}

The theory of planned behavior may explain why individuals when given certain opportunities, pressures, and reasons, act on their motivation to cheat when they believe the can get away with it. A person's ethical code has a large part to play in deciding to follow through with this behavior. Encouraging ethical decisions and reinforcing positive behaviors begins with the administration and faculty. There is no single solution for minimizing the risk of $\mathrm{AD}$, but as demonstrated in the studies of the TPB, there are foundational behaviors and motivations that if addressed in advance may significantly diminish the probability of this happening. TPB helps us to understand the probability that students will cheat given certain factors are present, permitting us to predict how they will perform in each situation. What can be done by universities to encourage ethical behaviors for students? Foremost, developing a culture of integrity and taking steps to eliminate many of the opportunity's students have to cheat. To dissuade students from unethical behaviors, universities can implement "zero tolerance policies" toward unethical behavior and have persistent reiteration of this expectation. Also, another component to address would be to mitigate some of the pressure's students perceive that prompt students to cheat. Perhaps through creating an infrastructure of support services which could address the personal pressures that today's students face such as work-school balance among others. The overall academic climate at an institution can be critical in the effectiveness of academic integrity by limiting opportunities for AD which can include strict adherence to policy, use of honor codes and a sense of ethical intellectual purpose. 


\section{RECOMMENDATIONS}

What can we do to reduce the amount of AD on college campuses? Two avenues are suggested the classroom environment and the campus environment.

\section{Classroom Atmosphere}

1. Review academic Integrity policy as part of the initial course orientation on the first day of class. During the next class meeting have the students complete a quiz based upon this policy.

2. Expanded use of essay examinations and questions. Research indicates that the only commonly used deterrent for cheating on examinations that is consistently effective is the practice of essay questions

3. Random seating for examinations. Since students tend to sit near their friends or who they know as "top students" not allowing students to sit in their usual seats during the examination increases the likelihood of reduced AD.

4. When creating an exam, faculty should randomize the selection of exam questions as well as the order in which they appear. The result is that students are not likely to get the same questions in the same sequence when taking an exam. This is also relevant if faculty allow students to repeat the exam. Each time this occurs, an exam will be made up of questions that are randomly selected and ordered.

5. Rather than using a fixed number of items that remain unchanged for each administration of the test, create a question pool. Questions can be grouped by any number of criteria, including topic, subject matter, question type or difficulty of question. A pool will generate an assessment with randomized questions selected by the faculty member. Pools can be created from new questions or questions in existing tests or pools. Pools are most effective when there are large numbers of questions in one group.

6. When creating an exam, faculty should randomize the selection of exam questions as well as the order in which they appear. The result is that students are not likely to get the same questions in the same sequence when taking an exam. This is also relevant if faculty allow students to repeat the exam. Each time this occurs, an exam will be made up of questions that are randomly selected and ordered.

\section{Campus Atmosphere}

The overall academic climate on campus can be critical. There must be some common sense of academic integrity that pervades on the campus.

1. Faculty should include a policy statement regarding academic integrity in their course syllabus. While this, in and of itself, may not be sufficient to change behavior, its acknowledged presence in the syllabus recognizes a commitment to honesty in the academic arena and establishes the clear expectation that academic integrity is an important principle to live by.

2. A campus-wide forum for entering students to acclimate themselves to the culture of academic integrity.

3. A required academic integrity course for all entering students.

4. Faculty and Administration should apply on a consistent basis all disciplinary procedures noted in the code of academic integrity. 


\section{LIMITATIONS AND FUTURE RESEARCH}

There are limitations of this study. This study only includes students from undergraduate colleges of business. The sample was taken from only two college of business student bodies in the southeastern portion of the United States. These conditions limit the generalizability of the results to other types of institutions throughout the United States and globally. Future research should include graduate and undergraduate students from all disciplines within a university. Studies across disciplines and that include larger samples utilizing diverse geographical locations throughout the United States and globally would better identify the breadth and depth of this problem.

\section{CONCLUSION}

In this paper, we have examined that $\mathrm{AD}$ is a crucial issue that universities need to confront. Educators and their universities have a responsibility to ensure that their students meet the challenging ethical standards in the global business environment. Universities must acknowledge that this is a problem that is not going away in the near future. It must place significant human and financial resources towards this issue. The implementation of academic integrity policies and procedures along with placing significant human and financial resources will empower a quality educational institution to uphold a high level of academic integrity. Adhering to this culture will prepare students to enter the global business environment with the professional ethical behaviors expected by future employers.

\section{REFERENCES}

Alschuler, A.S., \& Blimling, G.S. (1995). Curbing Epidemic Cheating Through Systematic Change. College Teaching, 123-127.

Ajzen, I. (1991). The Theory of Planned Behavior. Organizational Behavior and Human Decision Processes, 50, 179-211.

Beck, L., \& Ajzen, I. (1991). Predicting Dishonest Actions Using the Theory of Planned Behavior. Journal of Research in Personality, 25, 285-301.

Bowers, W. J. (1964). Student Dishonesty and Its Control in College. New York: Columbia University.

Bristor, J., \& Burke, M. (2016). Academic Integrity Policies: Has Your Institution Implemented an Effective Policy? The Accounting Educators' Journal, 1-10.

Carpenter, D., Harding, T., \& Finelli, C. (2010). Using Research to Identify Academic Dishonesty Deterrents Among Engineering Undergraduate. International Journal of Engineering Education, 26(5), 1156-165, 201.

Cronan, T. P., Mullins, J. K., \& Douglas, D. E. (2018). Further Understanding Factors that Explain Freshman Business Students' Academic Integrity Intention and Behavior: Plagiarism and Sharing Homework." Journal of Business Ethics, 147, 197-220.

Crown, D. E., \& Spiller, M. S. (1998). Learning from The Literature on Collegiate Cheating: A Review of Empirical Research. Journal of Business Ethics, 17, 683-700.

Desplaces, D.E., Melchar, D.E., Beauvais, L.L., \& Bosco, S.M. (2007). The Impact of Business Education on Moral Judgment Competence: An Emprical Study. Journal of Business Ethics, 74, 73-87.

Elias, R. Z. (2015). The Effect of Machiavellianism on Business Students' Perception of Cheating. Academy of Education Leadership Journal, 19(1), 175-183.

Etter, S., Gramer, J., \& Finn, S. (2006). Origins of Academic Dishonesty: Ethical Orientations and Personality Factors Associated with Attitudes about Cheating with Information Technology. Journal of Research on Technology in Education, 39(2), 133-155.

Fishbein, L. (1994). We Can Curb College Cheating. The Education Digest, 58-61. 
Harding, T. S., Carpenter, D. D., Finelli, C. J., \& Passow, H. J. (2004). Does Academic Dishonesty Relate to Unethical Behavior in Professional Practice? An Exploratory Study. Science and Engineering Ethics, 10(2), 1-14.

Hisam, C. K., Kassim, K., Emilina, N., Nasir, M., \& Ahmad, S. (2015). Academic Dishonesty of Accounting Students at Higher Learning Institutions. Mediterranean Journal of Social Sciences Rome-Italy, 6(4), 702-707.

Jackson, C. J., Levine, S. Z., Furnham, A., \& Burr, N. (2002). Predictors of Cheating Behavior at A University: A Lesson from the Psychology of Work. Journal of Applied Social Psychology, 32, 1031-1046.

Jones, D. L. (2011). Academic Dishonesty: Are More Students Cheating? Norfolk State University Business Communication Quarterly, 144.

Kerhvliet, J. (1994). Cheating by Economics Students: A Comparison of Survey Results. The Journal of Economic Education, 121-133.

McCabe, D. L., Butterfield, K. D., \& Trevino, L. K. (2006). Academic Dishonesty in Graduate Business Programs: Prevalence, Causes, and Proposed Action. Academy of Management Learning and Education, 5(3), 294-305.

McCabe, D. L. (2005). It Takes a Village: Academic Dishonesty and Educational Opportunity. Association of American Colleges and Universities, 91(3), 1-8.

McCabe, D. L., \& Trevino, L. K. (1997). Individual and Contextual Influences on Academic Dishonesty: A Multicampus Investigation. Research in Higher Education, 3(38), 379-396.

McCabe, D. L., \& Trevino, L. K. (1993). Academic Dishonesty: Honor Codes and Other Contextual Influences. The Journal of Higher Education, 64(5), 522-538.

McCabe, D. L., \& Trevino, L. K., \& Butterfield, K. D. (2001). Dishonesty in Academic Environments. The Journal of Higher Education, 72, 29-45.

Michaels, J. W., \& Miethe, T. D. (1989). Applying Theories of Deviance to Academic Cheating. Social Science Quarterly, 70, 870-885.

Nickerson, C., Schwarz, N., Diener E., \& Kahneman, D. (2003). Zeroing on the Dark Side of the American Dream: A Closer Look at the Negative Consequences of the Goal for Financial Success. Psychological Science, 14(6)6, 531-536.

Payne, S. L., \& Nantz, K. S. (1994). Social Accounts and Metaphors about Cheating. College Teaching, 90-96.

Perry, A. R., Kane, K. M., Bernesser, K.J., \& Spicker, P.T. (1990). Type-A Behavior, Competitive Achievement-Striving and Cheating Among College Students. Psychological Reports, 66, 459465.

Salwa, H., Ismail, Y., \& Ismail, S. (2018). Academic Dishonesty Among Accounting Students in Malaysia. Management and Accounting Review, 17(1).

Simha, A., \& Cullen, J. B. (2012). A Comprehensive Literature Review on Cheating. International Journal of Cyber Ethics in Education, 2(4), 24-44.

Stevens, G., \& Stevens, F. (1987). Ethical Inclinations of Tomorrow's Managers Revisited: How and Why Students Cheat. The Journal of Education for Business, 24-29.

Tom, G., \& Borin, N. (1988). Cheating in Academe. The Journal of Education for Business, 153-157.

Weimer, M. (2018). Cheating: Can We Be Doing More to Promote Academic Integrity? Higher Ed Teaching and Learning,.1-3.

Whitley, B. E. (1998). Factors Associated with Cheating Among College Students: A Review. Research in Higher Education, 39, 235-274.

Wilhoit, S. (1994). Helping Students Avoid Plagiarism. College Teaching, 161-164.

104 Journal of Leadership, Accountability and Ethics Vol. 17(1) 2020 